\title{
Research on Ubiquitous Map Visualization for Ternary Space
}

\author{
Si Wang, Guangxia Wang \\ Institute of Geographical Spatial Information, Information Engineering University, Zhengzhou, China \\ Email: plaieu_wangs@163.com
}

How to cite this paper: Wang, S., \& Wang, G. X. (2022). Research on Ubiquitous Map Visualization for Ternary Space. Journal of Geoscience and Environment Protection, 10, 162-170.

https://doi.org/10.4236/gep.2022.102010

Received: February 13, 2022

Accepted: February 25, 2022

Published: February 28, 2022

\begin{abstract}
In the era of information and communication technology (ICT) and big data, the map gradually shows a new qualitative feature of "spatiotemporal ubiquitous" with the extension of its object space, expression space and information source, which challenges the theory of cartographic visualization. This paper discusses the ubiquitous map visualization from the object content and expression form. Oriented to the ternary space, it divides the object dimension of ubiquitous map visualization and analyzes the expression characteristics of ubiquitous map visualization. Based on that, it constructs the variable system, symbol system and method system of ubiquitous map visualization. With three cases of the metro roadmap, the tag map, and the three-dimensional (3D) city map, the application of the proposed content is explained to illustrate its effectiveness. The research in this paper is expected to further enrich the theoretical basis of cartographic visualization and provide theoretical support for the expression and application of ubiquitous map visualization.
\end{abstract}

\section{Keywords}

Ubiquitous Map, Ternary Space, Visualization, Variables, Map Symbol, Scene

\section{Introduction}

As the core of map expression, visualization has always been one of the core contents of cartographic research. Traditional maps are oriented to the dual space of "geography-humanity" and use the form of "two-dimensional, static, graphical, and complete map structure" to visually express specific themed geographic scenes such as the spatial location distribution, connection, development and change status of geographic elements. In the era of ICT (information and communication technology) and big data, with the expansion of the object and expression space of maps from the traditional dual space of "geography-humanity" 
to the ternary space of "geography-humanity-information", and the expansion of information source of maps from standard geographic information to fragmented and multi-modal spatiotemporal ubiquitous information (Kresse \& Danko, 2012), maps gradually show the development trend of "ubiquitous", and the concept of "ubiquitous map" has also been proposed (Liu, Guo, Guo, Gao, \& Cui, 2020). Compared with traditional maps, the object content and expression form of ubiquitous map visualization are greatly expanded: in terms of object content, it is oriented to complex, diverse and dynamic scenes in the ternary space, expanding new expression content such as the network behavior of ubiquitous cyberspace, semantic information of collection space, etc. (Ai, 2016); in terms of expression form, driven by ICT and big data technologies, various new visualization forms are produced such as cyber maps, metaphor maps, semantic maps, we-maps, text maps, VR (virtual reality)/AR (augmented reality)/MR (mix reality) maps, etc.

New changes and expansions make the traditional map theory framework unable to provide sufficient support for ubiquitous map visualization, and map visualization research faces new challenges. On the one hand, compared with the enrichment of technical means, the development of the corresponding guiding theory is relatively lagging behind. Although the research framework, dimension division, and new content and form of ubiquitous map visualization have been preliminarily discussed (Guo, Chen, Ying, Lv, \& Li, 2018; Ai, 2016), the research at the theoretical level still needs to be enriched and deepened. On the other hand, the process of ubiquitous map visualization has broadened and introduced more diverse content objects, variable types and symbolic forms (Guo, Chen, Ying, Lv, \& Li, 2018; Ai, 2016; Ai, 1998; Gao, Zhu, Ying, \& Xiao, 2005; Jiang, Nie, \& Cao, 2009; Yang, Wu, \& Yang, 2021; Bai, Yan, Lu, Zhang, \& Zhang, 2021), the map representation has been greatly extended (Chen, 2020), and the existing cartographic theories have not yet formed a relatively complete visual expression system for this.

In view of the above, this paper discusses the ubiquitous map visualization in terms of object content and expression form. Oriented to the ternary space, it divides the object dimension of ubiquitous map visualization, analyzes the expression characteristics of ubiquitous map visualization, and presents the variable system, symbol system and method system of ubiquitous map visualization. The research in this paper is hoped to expand the theoretical basis of traditional map visualization and provide theoretical support for the expression and application of ubiquitous map visualization in ternary space.

\section{Ternary Space and Ubiquitous Map Visualization}

\subsection{Ternary Space and Ubiquitous Map}

The concept of "ternary space" refers to the ternary space of "geography-humanity-information" formed by the deep integration of geographic space, humanity space and information space. "Geographic space" refers to the 
natural environment and material systems that human beings rely on for survival, including various natural geographical elements and natural geographical scenes. "Humanity space" refers to the human social world, including human behavior, humanistic networks, social activities, and social processes. "Information space" refers to the cyber world, which covers the ubiquitous network composed of various communication networks, the Internet, the Internet of Things and professional sensor networks, and the data information acquired, recorded, transmitted and processed with the "ubiquitous" technical characteristics. In the information space, there are things such as network nodes, server nodes, DNS, etc., and there occur network events, phenomena and processes such as information exchange, click access, upload and download, and data transmission.

The ubiquitous map is an abstract description and comprehensive expression of ternary space under the support of map projection, map synthesis, and map visualization (Wang, Wang, \& Tian, 2021), which analyzes, refines and describes the features of ternary space objects by means of map language, image thinking and spatial thinking, and realizes functions such as information acquisition, transmission, and cognition between people and people, people and things, and things and things (Guo, Chen, Ying, Lv, \& Li, 2018). From the dual space of "geography-humanity" to the ternary space of "geography-humanity-information", the expression space of the map is no longer only the informatization of natural space and human space, but also contains the new map expression space expansion brought by information space, including cyberspace, virtual space, micro-small space, collection space, social media space, social psychological space, etc. $(\mathrm{Ai}, 2016)$. The things, phenomena, processes and even non-spatial information with pure semantic features that occur or exist in these spaces can be simulated and expressed in a visual form with the help of ubiquitous maps.

\subsection{Object Dimension Division}

Dimension is the fundamental quantity of object expression. For the ternary space content, according to the theory of scene science ( $\mathrm{Lv}, \mathrm{Yu}, \mathrm{Yuan}, \mathrm{Luo}$, Zhou, Wu et al., 2018) and the idea of ubiquitous map information division (Wang, Wang, \& Tian, 2021), ubiquitous map visualization objects can be divided into six dimensions: time dimension, place dimension, person dimension, thing dimension, event dimension and phenomenon dimension (Table 1). Each dimension actually represents an aspect of the ubiquitous map visualization content and can be represented by features with different levels of refinement. The combination and association of different dimensions form diverse content scenes, that is, a complex with specific structures and functions formed by the interconnection and interaction of various elements in different ranges in the ternary space (Lv, Yu, Yuan, Luo, Zhou, Wu et al., 2018). The dimensional division is the abstraction, integration and induction of complex elements, information, and data in ternary space for the needs of map visualization. In theory, ternary space objects of any granularity or type can be divided into these six dimensions. 
Table 1. Visualization elements of different dimensions.

\begin{tabular}{ll}
\hline Dimensions & Visualization Elements \\
\hline time & date, time point, time range, temporal relationship \\
place & place name information, address, website, coordinates, spatial point, spatial range, spatial relationship \\
person & name, hobby, profession, granularity (individual, group, organization), role, character relationship \\
thing & name, nature, composition, relationship of things \\
event & name, theme, nature, impact, cause, process, result, time of occurrence, place of occurrence, participants, intensity \\
phenomenon & name, theme, nature, impact, intensity, evolutionary process, condition \\
\hline
\end{tabular}

\subsection{Characteristics of Ubiquitous Map Visualization}

The ternary space greatly expands the expression content of ubiquitous map visualization, and makes its expression form break through the constraints of traditional physical space. For ternary space, ubiquitous map visualization shows the following characteristics:

1) Intuitiveness and figurativeness. Ubiquitous map visualization can intuitively and vividly display ternary space object information, reflect the characteristics of big data, and simulate dynamic changes through a variety of map languages such as graphics, network diagrams, images, tags, animations and sounds.

2) Integration and diversification. Ubiquitous map visualization integrates multimedia technologies of text, graphics, images, sounds, animations and videos, and can comprehensively express ternary space information in multiple forms, multiple perspectives, multiple senses, and multiple levels.

3) Interactivity and dynamics. Ubiquitous map visualization can realize dynamic and real-time interaction between humans and machines in terms of vision, hearing, and even touch through technologies such as virtual reality, augmented reality, mixed reality, 3D simulation, and computer animation.

4) Pattern view. Compared with traditional maps, ubiquitous map visualization pays more attention to the representation of information connotations and relationships, pursuing a pattern view. In order to highlight key information, ubiquitous map visualization can allow the mapping method of magnifying distortion and deformation and reduce the requirements for positioning accuracy and scalability.

\section{Ubiquitous Map Visualization Method}

\subsection{Variable System}

Variables are the basis of ubiquitous map visualization, and are used to describe the content features of ternary space objects such as space-time, attributes, and semantics. The variables of traditional map visualization mainly refer to J. Bertin visual variables including shape, size, direction, color, brightness, and density, which show the characteristics of two-dimensional and static. In the ternary space, the variables of ubiquitous map visualization have been greatly expanded 
in terms of sense, geometry, state, language form, etc., further containing hearing variables, touch variables, dynamic variables, three-dimensional variables, text variables, and image variables. This paper summarizes and integrates them to form a variable system of ubiquitous map visualization (Table 2).

\subsection{Map Symbol System}

Map symbols are the core of ubiquitous map visualization. They are composed of visualization variables to refer to and reflect the content of ternary space objects such as time, place, person, thing, event, and phenomenon. Under the ternary space, the ubiquitous map visualization symbol design is very personalized, diverse and flexible. With the support of rich visualization variables and advanced information technologies, there are more diverse choices in sensory, spatial geometry, abstract form, state, style, and expression language, such as visual, hearing, touch, two-dimensional (2D), three-dimensional (3D), point, line, area, dynamic, static, machine-painted, hand-painted, graphics, characters, etc., forming new types such as animation symbols, 3D symbols, tag symbols, etc. This paper analyzes and summarizes them, and initially establishes a ubiquitous map visualization symbol system (Table 3 ). In Table 3, the “ $\sqrt{ }$ " indicates optional factors in the visualization design of specific types of map symbols.

\subsection{Scene-Oriented Visualization Method System}

Rich visualization variables, flexible symbol design, and the support of multimedia, 3D simulation and other technologies have formed a variety of ubiquitous map visualization forms and methods, such as cyber network maps, text maps, $3 \mathrm{D}$ virtual geographic environments, and hand-painted maps, metaphor maps, etc. These methods have their own characteristics and have their own advantages in expressing ternary space objects and scenes. It is worth noting that no single visualization method is suitable for all cartographic objects and scene requirements, and the choice of visualization methods should depend on object characteristics and scene content. In this regard, this paper establishes a scene-oriented visualization method system of ubiquitous maps (Table 4).

Table 2. Visualization variable system.

\begin{tabular}{|c|c|c|}
\hline \multicolumn{2}{|c|}{ Variable System } & Metrics \\
\hline \multirow{5}{*}{$\begin{array}{c}\text { visual } \\
\text { variables }\end{array}$} & $2 \mathrm{D}$ static visual variable & shape, size, direction, color, brightness, density \\
\hline & dynamic visual variable & duration, change rate, change order, rhythm \\
\hline & $3 \mathrm{D}$ visual variables & pose, texture, lighting, shadow, clarity, styling \\
\hline & image visual variables & image elements \\
\hline & text visual variables & font, font size, font color, font type, font column, font spacing, font direction, font position \\
\hline \multicolumn{2}{|c|}{ hearing variables } & loudness (volume), frequency, duration, sound source location, timbre, pleasantness \\
\hline \multicolumn{2}{|c|}{ touch variables } & touch strength, vibration frequency, vibration time, touch point \\
\hline
\end{tabular}


Table 3. Visualization map symbol system.

\begin{tabular}{|c|c|c|c|c|c|c|c|c|c|c|c|c|c|c|}
\hline \multirow{2}{*}{ Map Symbol Type } & \multicolumn{3}{|c|}{ Sense } & \multicolumn{2}{|c|}{$\begin{array}{c}\text { Spatial } \\
\text { Geometry }\end{array}$} & \multicolumn{3}{|c|}{ Abstract Form } & \multicolumn{2}{|c|}{ State } & \multicolumn{2}{|c|}{ Style } & \multicolumn{2}{|c|}{$\begin{array}{l}\text { Expression } \\
\text { Language }\end{array}$} \\
\hline & Visual & Hearing & Touch & $2 \mathrm{D}$ & $3 \mathrm{D}$ & Point & Line & Area & Static & Dynamic & $\begin{array}{l}\text { Machine- } \\
\text { painted }\end{array}$ & $\begin{array}{l}\text { Hand- } \\
\text { painted }\end{array}$ & Graphic & Character \\
\hline animated symbol & $\sqrt{ }$ & $\sqrt{ }$ & & $\sqrt{ }$ & $\sqrt{ }$ & $\sqrt{ }$ & $\sqrt{ }$ & $\sqrt{ }$ & & $\sqrt{ }$ & $\sqrt{ }$ & & $\sqrt{ }$ & \\
\hline 3D symbol & $\sqrt{ }$ & & & & $\sqrt{ }$ & & & & $\sqrt{ }$ & $\sqrt{ }$ & $\sqrt{ }$ & $\sqrt{ }$ & $\sqrt{ }$ & \\
\hline tag symbol & $\sqrt{ }$ & & & $\sqrt{ }$ & & $\sqrt{ }$ & & $\sqrt{ }$ & $\sqrt{ }$ & & $\sqrt{ }$ & & & $\sqrt{ }$ \\
\hline node symbol & $\sqrt{ }$ & & & $\sqrt{ }$ & $\sqrt{ }$ & $\sqrt{ }$ & & & $\sqrt{ }$ & & $\sqrt{ }$ & & $\sqrt{ }$ & \\
\hline directed line symbol & $\sqrt{ }$ & & & $\sqrt{ }$ & $\sqrt{ }$ & & $\sqrt{ }$ & & $\sqrt{ }$ & & $\sqrt{ }$ & & $\sqrt{ }$ & \\
\hline hot spot symbol & $\sqrt{ }$ & & & $\sqrt{ }$ & & $\sqrt{ }$ & & & $\sqrt{ }$ & $\sqrt{ }$ & $\sqrt{ }$ & & $\sqrt{ }$ & \\
\hline image symbol & $\sqrt{ }$ & & & $\sqrt{ }$ & $\sqrt{ }$ & & & $\sqrt{ }$ & $\sqrt{ }$ & & $\sqrt{ }$ & & $\sqrt{ }$ & \\
\hline hand-painted symbol & $\sqrt{ }$ & & & $\sqrt{ }$ & $\sqrt{ }$ & $\sqrt{ }$ & $\sqrt{ }$ & $\sqrt{ }$ & $\sqrt{ }$ & & & $\sqrt{ }$ & $\sqrt{ }$ & \\
\hline blind map symbol & & $\sqrt{ }$ & $\sqrt{ }$ & & $\sqrt{ }$ & $\sqrt{ }$ & $\sqrt{ }$ & $\sqrt{ }$ & $\sqrt{ }$ & & $\sqrt{ }$ & & $\sqrt{ }$ & $\sqrt{ }$ \\
\hline
\end{tabular}

Table 4. Scene-oriented visualization method system.

\begin{tabular}{|c|c|c|}
\hline \multicolumn{2}{|c|}{ Visualization Methods } & \multirow{2}{*}{$\begin{array}{l}\text { Content and Application Scene } \\
\text { node links, online/offline, and accessibility in cyberspace }\end{array}$} \\
\hline topological & cyber network map & \\
\hline structure & schematic map & connectivity between sites or nodes \\
\hline & cartogram & quantitative differences between regions \\
\hline \multirow{2}{*}{$\begin{array}{l}\text { human-computer } \\
\text { interaction and } \\
\text { multi-angle }\end{array}$} & semantic map & navigation and positioning, semantic information acquisition \\
\hline & $\begin{array}{l}3 \mathrm{D} \text { virtual geographic } \\
\text { environment }\end{array}$ & $\begin{array}{l}\text { 3D geometric information and semantic information of different levels of detail about } \\
\text { ground objects, supporting geographic simulation calculation or information } \\
\text { transmission for smart cities }\end{array}$ \\
\hline \multirow{3}{*}{$\begin{array}{l}\text { organization of } \\
\text { big data }\end{array}$} & multi-slice image map & multi-angle and multi-level 3D surface information and object status \\
\hline & text map & $\begin{array}{l}\text { organization and expression of massive and unstructured geographic text data for } \\
\text { public opinion analysis, city portraits, advertising, etc. }\end{array}$ \\
\hline & hot spot map & $\begin{array}{l}\text { organization and expression of incremental data streams to reflect the semantic and } \\
\text { location information of high-attention elements }\end{array}$ \\
\hline & migration map & trajectory and characteristics of population migration \\
\hline $\begin{array}{l}\text { semantic } \\
\text { spatialization }\end{array}$ & metaphor map & ontological characteristics such as the composition, nature, type, and efficacy of objects \\
\hline \multicolumn{2}{|c|}{ artistic rendering hand-painted map } & $\begin{array}{l}\text { unique cultural themes, historical and cultural information for tourism, advertising, } \\
\text { etc. }\end{array}$ \\
\hline & map animation & movement changes of ternary space objects, emphasis enhancement \\
\hline
\end{tabular}

\section{Example and Analysis}

The following takes the metro roadmap, tag map and 3D city map as examples to analyze the application of the ubiquitous map visualization content, variable system, symbol system and method system proposed in this paper. 
The metroroadmap is a kind of schematic map. It is a visual scene expression with the theme of subway traffic phenomena. It mainly focuses on the topological information content such as subway stations and the connectivity between stations. In visualization, it adopts the topological structure, including node symbols, connection line symbols, and annotation symbols. Among them, node symbols and connection line symbols are composed of shape and color variables, and annotation symbols are composed of text variables such as font, font size, font color, font direction, and font position (see Figure 1).

The tag map is an emerging form of map visualization that associates a series of text tags with their expressed spatial locations, and displays the tag cloud composed of these text labels in the relevant area on the map (Yang, Wu, \& Yang, 2021). With the help of tag maps, users can quickly and accurately extract, compare, analyze, and express key information about the subject content of regional texts. The visualization of the tag map is mainly based on tag symbols, and the visualization variables mainly include font, font size, font color, font type, font column, font spacing, font direction and font position (see Figure 2).

$3 \mathrm{D}$ city map is a specific application of $3 \mathrm{D}$ virtual geographic environment for cities, focusing on the geometric correspondence between 3D city and the real world at different levels of detail and the expression of semantic information. The visualization of the 3D city map is mainly based on 3D map symbols, supplemented by annotation symbols. Among them, 3D map symbols are composed of visual variables such as pose, texture, lighting, shadow, clarity, and styling; annotation symbols are composed of text visual variables such as font color, font, and font position (see Figure 3).

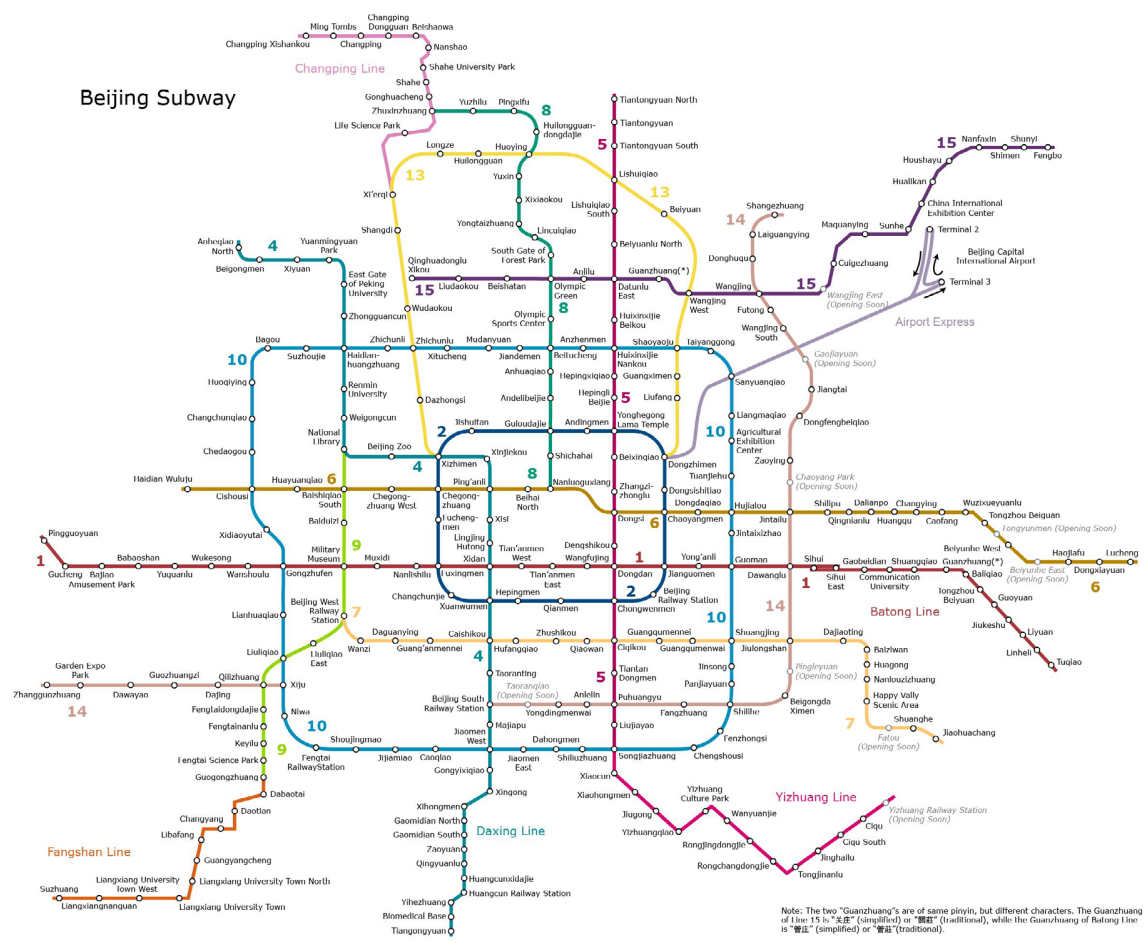

Figure 1. Metro roadmap (from https://image.baidu.com). 


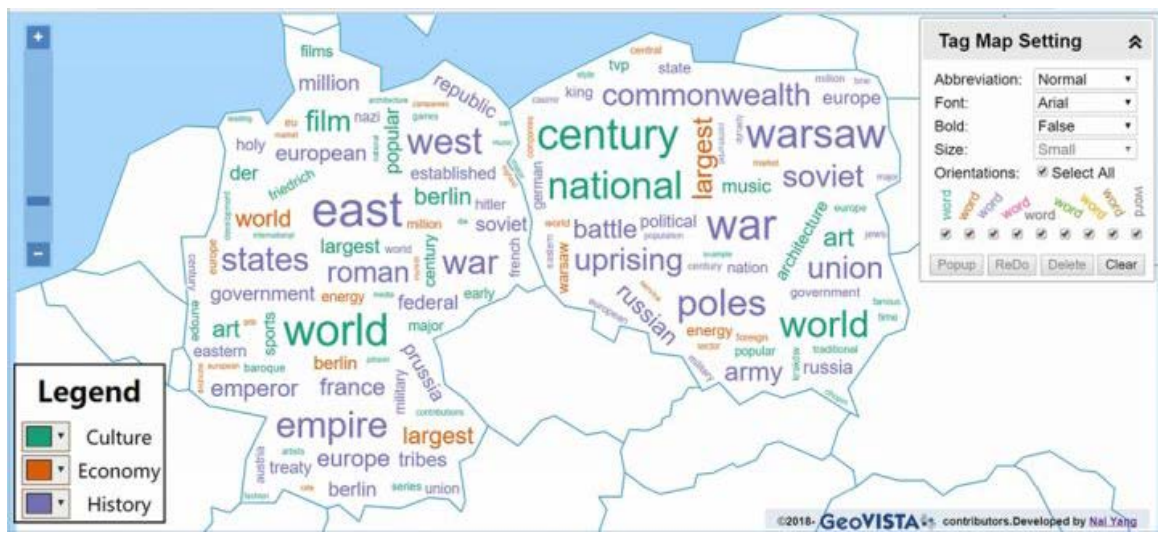

Figure 2. Tag map (from https://xgxy.cug.edu.cn/info/1031/2549.htm).

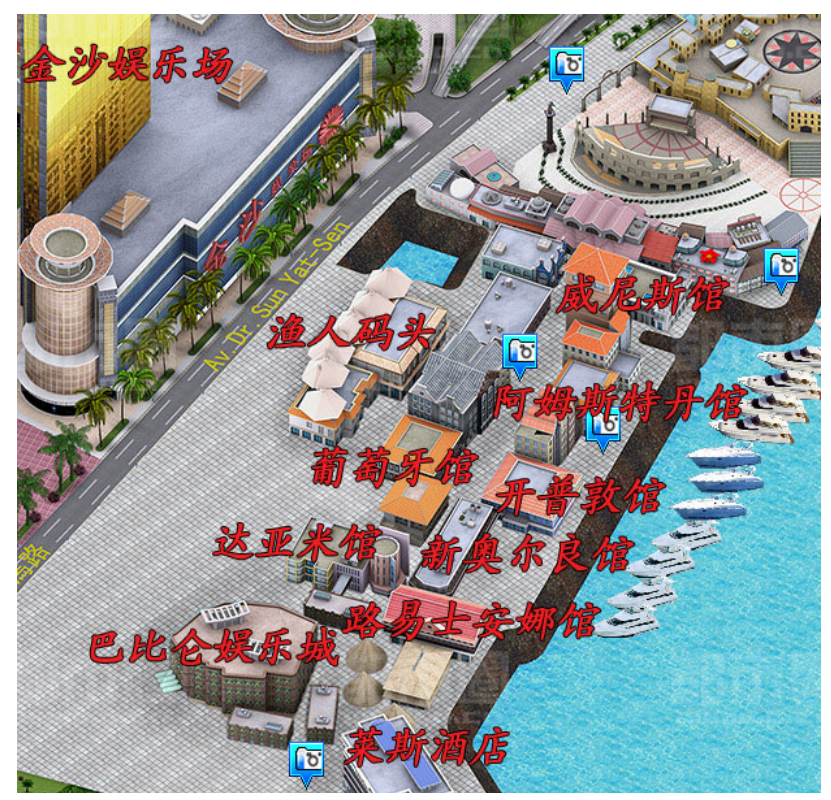

Figure 3. 3D city map (from http://www.onegreen.net/).

\section{Conclusion}

In the era of ICT and big data, with the expansion of map object and expression space from the dual space of "geography-humanity" to the ternary space of "geography-humanity-information", and the expansion of information source from standard geographic information to multimodal and fragmented spatiotemporal ubiquitous information, the concept of "ubiquitous map" emerges as the times require. The addition of information space greatly expands the object content and expression of ubiquitous map visualization, and the traditional map visualization theory faces unprecedented challenges. Facing the ternary space, this paper studies ubiquitous map visualization from the perspective of object content and expression form, forms the object dimension division of ubiquitous map visualization, and establishes the variable system, symbol system and method system of ubiquitous map visualization, and realizes the preliminary expansion of the theoretical basis of traditional map visualization. The variable, 
symbol and method systems proposed in this paper are extensible, and their contents will be continuously expanded and refined with the development of visualization theory and technology.

\section{Conflicts of Interest}

The authors declare no conflicts of interest regarding the publication of this paper.

\section{References}

Ai, T. H. (1998). Dynamic Symbol and Dynamic Map. Journal of Wuhan Technical University of Surveying and Mapping, 23, 47-51.

Ai, T. H. (2016). Development of Cartography Driven by Big Data. Journal of Geomatics, $41,1-7$.

Bai, Y. L., Yan, H. W., Lu, X. M., Zhang, X. G., \& Zhang, J. (2021). Visual Variables of We-Maps Symbols and Their Applications. Science of Surveying and Mapping, 46, 182-188, 204.

Chen, Y. F. (2020). Quasi-Map System and Its Diagrammatic Construction. Thesis, East China Normal University.

Gao, Y. R., Zhu, Q., Ying, S., \& Xiao, J. P. (2005). Visual Variable of 3D Models for 3D GIS. Science of Surveying and Mapping, 30, 41-43.

Guo, R. Z., Chen, Y. B., Ying, S., Lv, G. N., \& Li, Z. L. (2018). Geographic Visualization of Pan-Map with the Context of Ternary Space. Geomatics and Information Science of Wuhan University, 43, 1603-1610.

Jiang, N., Nie, B., \& Cao, Y. N. (2009). The Pilot Study of Apperceive Variables in Animated Map. Geomatics World, 4, 29-32.

Kresse, W., \& Danko, D. M. (2012). Springer Handbook of Geographic Information. Springer. https://doi.org/10.1007/978-3-540-72680-7

Liu, J. N., Guo, W. F., Guo, C., Gao, K. F., \& Cui, J. S. (2020). Rethinking Ubiquitous Mapping in the Intelligent Age. Acta Geodaetica et Cartographica Sinica, 49, 403-414.

Lv, G. N., Yu, Z. Y., Yuan, L. W., Luo, W., Zhou, L. C., Wu, M. G. et al. (2018). Is the Future of Cartography the Scenario Science?. Journal of Geo-information Science, 20, 1-6.

Wang, S., Wang, G. X., \& Tian, J. P. (2021). Classification Model of Ubiquitous Map Information Facing Location-Based Aggregation. Acta Geodaetica et Cartographica Sinica, 50, 789-799.

Yang, N., Wu, G. J., \& Yang, C. C. (2021). Overview of Text Map Visualization. Journal of Geomatics, 46, 48-52. 